\title{
Detailed Analysis of the Intracavity Phenomena Inside a Cylindrical Microresonator
}

\author{
D. J. W. Klunder, Student Member, IEEE, M. L. M. Balistreri, F. C. Blom, H. J. W. M. Hoekstra, \\ A. Driessen, Senior Member, IEEE, L. Kuipers, and N. F. van Hulst
}

\begin{abstract}
Based on a rigorous analysis of the intensity distribution inside a cylindrical microresonator (MR), a detailed description of the wavelength and spatial dependence of the intracavity intensity is given. The theory is in accordance with photon scanning tunneling microscopy (PSTM) of an integrated optics MR. Good agreement between the theory and the PSTM measurements is found.
\end{abstract}

Index Terms-Intracavity phenomena, microresonator (MR), photon scanning tunneling microscopy (PSTM), waveguide theory.

\section{INTRODUCTION}

C YLINDRICAL microresonators (MRs) have attracted a lot of attention in recent research. They have been proposed as add-drop filter [1], [2], microlaser [3], [4], and all-optical switch [5], and they open the route to large-area reduction of complex integrated optics devices [6]. For all of these applications, specialized fabrication methods and detailed understanding of the MRs are crucial.

A common approach in the design of cylindrical MRs is to consider it as a black box and use a parametric model describing the functional behavior of the MR [1]. For characterizing the functional behavior of an MR, it is sufficient to measure the power in the straight waveguides (SWs) coupled with the MR, without considering the intensity distribution inside the MR [2], [7]. By this somewhat indirect way, only a single measurable quantity (i.e., the power in the SW) is obtained, which depends on all of the whispering gallery modes (WGMs) present in the MR and their respective coupling with the SW modes. One can deduce the effect of the WGMs on the power in the SW by a spectral analysis that yields the free spectral range (FSR) and finesse $(F)$. For a highly multimodal MR with closely spaced

Manuscript received June 20, 2001; revised September 24, 2001. This work was supported in part by the Stichting voor Fundamenteel Onderzoek der Materie (FOM) and the Nederlandse Organizatie voor Wetenschappelijk Onderzoek (NWO).

D. J. W. Klunder, H. J. W. M. Hoekstra, and A. Driessen are with the Lightwave Devices Group, MESA + Research Institute and Department of Applied Physics, University of Twente, Enschede 7500 AE, The Netherlands.

M. L. M. Balistreri was with the Applied Optics Group, MESA + Research Institute and Department of Applied Physics, University of Twente, Enschede 7500 AE, The Netherlands. He is now with Philips Research, Eindhoven 5056 AA, The Netherlands.

F. C. Blom was with the Lightwave Devices Group, MESA + Research Institute and Department of Applied Physics, University of Twente, Enschede 7500 AE, The Netherlands. He is now with JDS Uniphase Netherlands, Eindhoven 5056 AA, The Netherlands.

L. Kuipers and N. F. van Hulst are with the Applied Optics Group, MESA + Research Institute and Department of Applied Physics, University of Twente, Enschede $7500 \mathrm{AE}$, The Netherlands.

Publisher Item Identifier S 0733-8724(02)02189-8. (with respect to the FSR) WGMs, an extraordinarily large wavelength scan (a few hundred nanometers) is needed in order to resolve the WGMs present. In practice, such a large wavelength scan range is often not available and, as a consequence, these MRs cannot be characterized adequately in this way.

As an alternative, the intensity distribution inside the MR can be mapped. From measuring this intensity distribution, as a function of the position in the MR and the wavelength $(\lambda)$, not only the WGMs themselves can be observed directly [8], [9], but also the interference (i.e., spatial and spectral mode-beat phenomena) between them [8], [10], [11]. Conventional (diffraction limited, $\Delta x \geq \lambda / 2$ ) microscopes, which rely on collecting the power scattered from the MR, cannot provide detailed information about the transversal modal profiles of the WGMs because the lateral dimensions of the WGMs are in the subwavelength regime. In contrast, the spatial resolution of a photon scanning tunneling microscope (PSTM) is not limited by diffraction, but determined by the effective size of the aperture of the fiber tip [12]. As the PSTM probes the evanescent field, it is possible to image the intensity distribution, especially for smooth interfaces (i.e., no scattering from the MR). This opens the possibility for obtaining detailed spatial information about the intensity distribution inside optical waveguides [8]-[17].

In this paper, a parametric model of the intracavity intensity of a (multimodal) cylindrical MR is developed. From this model, expressions for the FSR, spatial mode-beat periods, and spectral mode-beat periods of the interference between the WGMs are derived. Simple estimates for the order of magnitude of intracavity phenomena like spatial and spectral mode beat are given. In Section II, the choice for a parametric model of the intracavity intensity inside an MR is motivated. A detailed discussion of the parametric model is given in Section III. In Section IV, the model (in conjunction with detailed simulation tools for calculating the WGMs) is applied to the analysis of intracavity phenomena in a highly multimodal cylindrical MR and compared with detailed PSTM measurements of the intracavity intensity.

\section{MOTIVATION}

In this paper, highly multimodal cylindrical MRs exhibiting a rich variety of intracavity phenomena are discussed. To illustrate the complex nature of the intracavity intensity inside a highly multimodal MR, we briefly discuss PSTM measurements of a highly multimodal MR. Fig. 1(a) shows an example of the spatial distribution of the intracavity intensity inside an MR measured with a PSTM. Simultaneously, the topography of the MR was obtained by the shear force feedback of the fiber tip, as shown in Fig. 1(b). In Fig. 1(a), one can clearly observe 


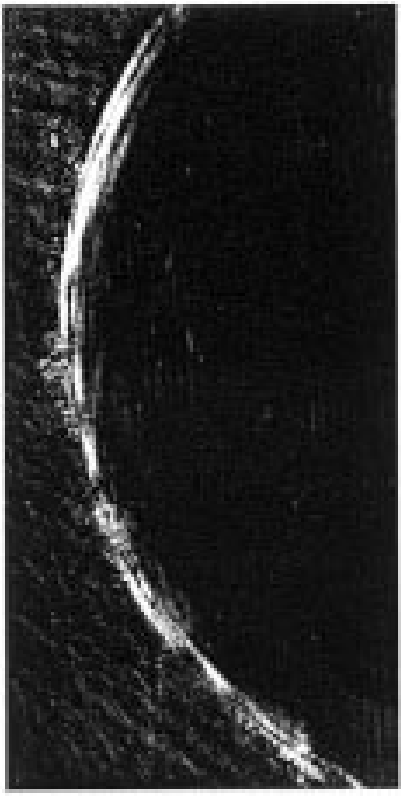

(a)

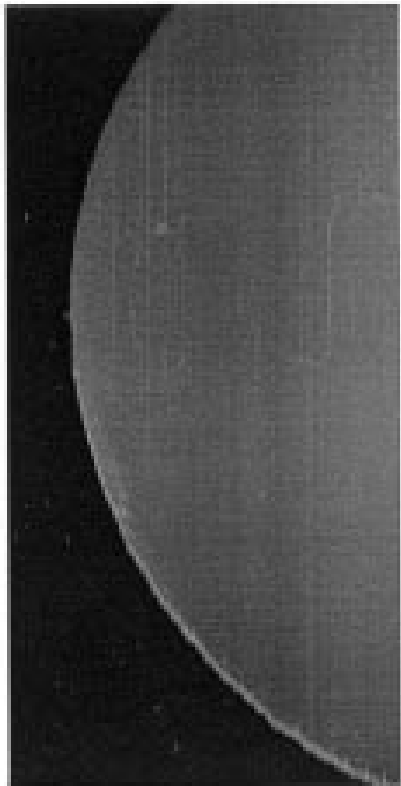

(b)
Fig. 1. (a) Close-up of the measured spatial distribution of the intensity inside a cylindrical MR. (b) Simultaneously measured topography of the cylindrical MR.

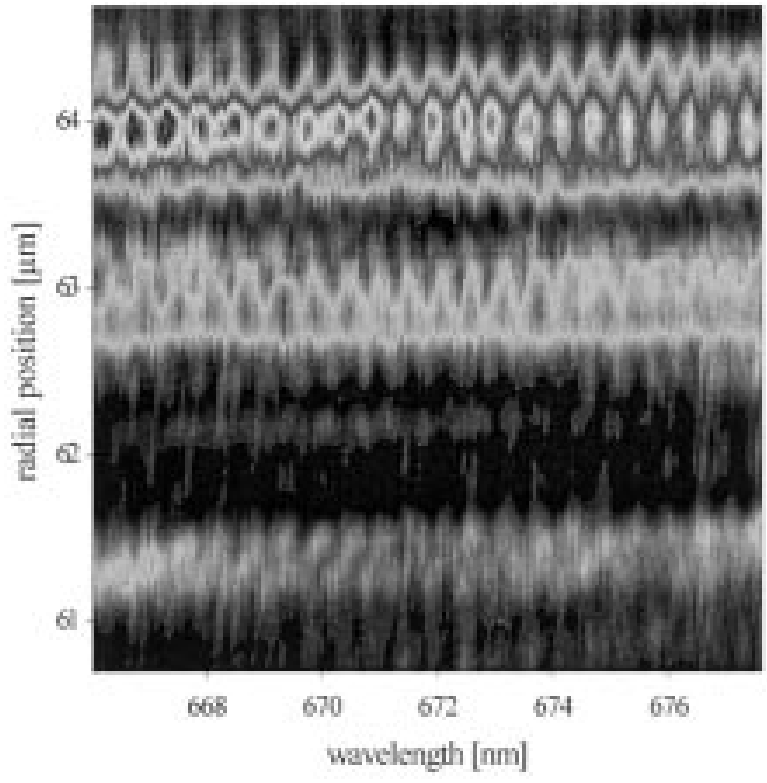

Fig. 2. Example of the measured spatiospectral scan of the intracavity intensity close to the rim of the cylindrical MR.

ring-shaped intensity profiles positioned close to the rim of the MR. The modulations on top of these intensity profiles exhibit the interference between the WGMs present in the MR. Fig. 2 shows an example of a spatiospectral scan of the intensity inside the MR at a fixed angle of observation. Three bands, exhibiting the on- and off-resonance behavior as a function of the wavelength, corresponding with different WGMs can be discerned.

From Figs. 1 and 2, it can be concluded that the intracavity intensity of a highly multimodal MR exhibits a rich variety of intracavity phenomena that cannot be modeled by a parametric model of the functional behavior of the MR only. By finite difference time domain (FDTD) simulations of cylindrical MRs
[18], the spatial and spectral (by applying a Fourier transformation with respect to the time) distribution of the intracavity intensity can be modeled rigorously, but the method is time consuming, especially for structures with dimensions larger than a few wavelengths, and additional analysis is needed for resolving the contributions of the individual WGMs. A parametric model of the intracavity intensity in terms of the modal profiles and propagation constants of the WGMs, which gives analytical expressions for experimental parameters like the spatial and spectral frequencies of the intracavity intensity, is expected to simplify the analysis of PSTM measurements considerably. Because the experimentally determined properties of the WGMs can directly be related to the functional behavior of the MR, a parametric model of the intracavity intensity in combination with PSTM measurements can give useful input for the design process and evaluation of simulation methods.

\section{PARAmetric Model of InTRACAVITy PHENOMENA}

In the following, a parametric model describing the intracavity phenomena of a cylindrical MR is developed. First, the basic characteristics of the cylindrical MR and the WGMs are reviewed (see Section III-A). In Section III-B, the intracavity intensity inside a cylindrical MR is discussed and expressions for the power collected by the PSTM are given. Based on the results of Section III-B, simple expressions describing a variety of intracavity phenomena with only the propagation constants of the WGMs as input parameters are discussed in Sections III-C-E. Finally, Section III-F discusses the magnitudes of the intracavity phenomena in terms of the propagation losses and the transversal modal profiles of the WGMs.

\section{A. Cylindrical MRs and Whispering Gallery Modes}

Consider a cylindrical MR with a radius $R$ coupled with a single monomodal SW [see Fig. 3(a)]. A small fraction of the input field is coupled into the MR. After having completed a round-trip in the MR, the field interferes with the fraction of the input field just coupled into the MR. For certain wavelengths, the interference is constructive and, consequently, the MR is in resonance. The field inside a disk resonator can be expressed as a linear combination of the WGMs present. These WGMs $\left(P_{q}\right)$ are described by their polarization state $P(\mathrm{TE} / \mathrm{TM})$, slab order $k$, radial order $l$, and angular propagation constant $\gamma_{q}(q=$ $k, l)$. Throughout this paper, we assume semivectorial continuous wave $(\mathrm{CW})$ fields with a time dependence $\exp (j \omega t)$. As a consequence, $\gamma_{q}$ is an element of the fourth quadrant of the complex plane, with the imaginary part corresponding to the propagation losses of the WGM

$$
\gamma_{q}=\gamma_{q}^{\prime}-j \gamma_{q}^{\prime \prime}
$$

In our analysis, we only account for the wavelength dependence of the real part of the angular propagation constant; the wavelength dependence of all other parameters is assumed to be constant.

\section{B. Model of the Intracavity Intensity}

The intracavity field in an MR can be expressed in terms of the WGMs propagating in the counterclockwise and clockwise 


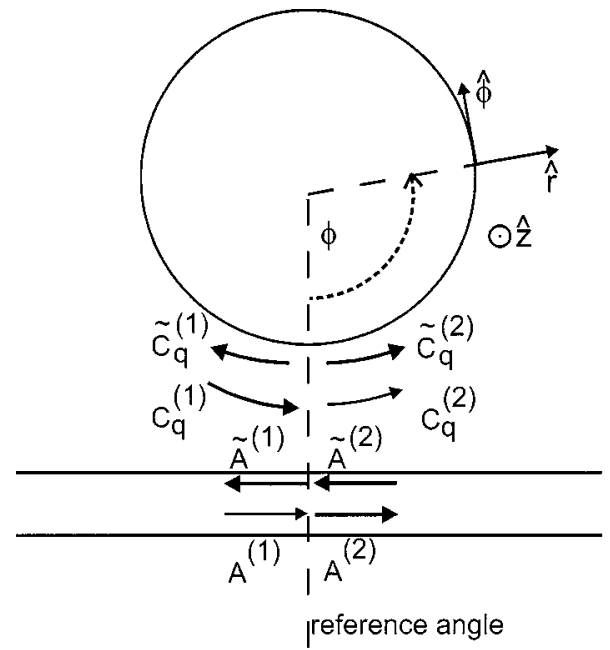

(a)

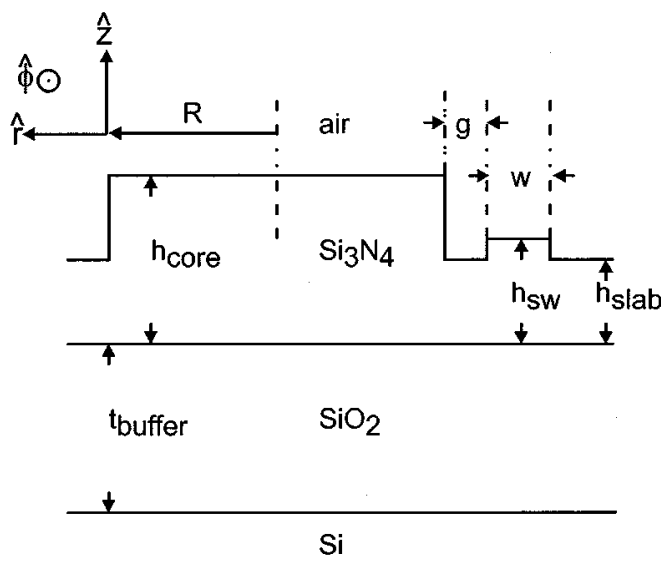

(b)

Fig. 3. (a) Schematic top view, using cylindrical coordinates, of a cylindrical MR coupled to a single SW and the modal amplitudes of the counterclockwiseand clockwise-propagating whispering gallery modes with respect to the reference angle. (b) Vertical cross section of the MR and SW at the reference plane. The MR $\left(h_{\text {core }}=293 \mathrm{~nm}, h_{\text {slab }}=166 \mathrm{~nm}\right.$ and $\left.R=64 \mu \mathrm{m}\right)$ is coupled with a SW ( $w=2.5 \mu \mathrm{m}$ and $\left.h_{\mathrm{sw}}=167 \mathrm{~nm}\right)$ at a distance (gap) $g=0.5 \mu \mathrm{m}$. The $\mathrm{Si}_{3} \mathrm{~N}_{4}$ layer was deposited on a $t_{\text {buffcr }}=3.2 \mu \mathrm{m}$ thick $\mathrm{SiO}_{2}$ layer in order to avoid leakage into the silicon substrate.

directions. As small reflections in the MR are enhanced due to the inherent feedback in an MR [19], [20], so should the contribution of contradirectionally propagating fields be incorporated into our model. Throughout this paper, it is assumed that the contradirectionally propagating fields are generated at the coupling between the SW and the MR and that no coupling between WGMs of different orders can occur. An extended model that does account for the generation of contradirectionally propagating WGMs inside the MR and the coupling between WGMs of different orders would be too involved and is beyond the scope of this paper [19], [20]. Backreflections from the in- and out-coupling facets of the straight input waveguide are ignored [i.e., $A^{\prime(2)}=0$ ]. The influence of the fiber probe (see also Section IV-B) on the intracavity is assumed to be small and, therefore, is not included in our model.

The excitation of the counterclockwise-propagating WGMs (modal amplitudes $C_{q}$ ) is realized by evanescent field coupling (with $\kappa_{q}, t_{q}$ defined as the evanescent field coupling coefficients between the SW mode and the codirectionally propagating WGMs) with the SW mode [modal amplitude $A^{(1)}$ ]. Because the coupling occurs in a small region around the point of closest distance between the MR and the SW [see Fig. 3(a)], we model it at the reference angle (see also [21]). In our model, we also include clockwise-propagating WGMs (modal amplitudes $C_{q}^{\prime}$ ) generated at the reference angle (i.e., $\phi=0$ ) due to the perturbation introduced by the SW, with $r_{q}, \Gamma_{q}$ as reflection coefficients describing the excitation of contradirectionally propagating WGMs $P_{q}$ by the SW mode, WGM $P_{q}$, respectively.

The fractions of the transverse electric (TE)- and transverse magnetic (TM)-polarized light collected by the PSTM no longer have mutually perpendicular polarizations inside the fiber probe and, as a consequence, a quasi-interference pattern between these originally mutually perpendicularly polarized fields can be observed [17]. Because we are primarily interested in the spatial and spectral dependence of the intracavity phenomena and less in the orders of magnitude of the phenomena, we assume that all of the WGMs are collected by the fiber probe with the same efficiency (see also Section IV-B) and that the field in the fiber is proportional with a scalar superposition $\left(E=E_{f}\right)$ of the TE- and TM-polarized WGMs at the aperture $\left(z=d_{\text {tip }}\right)$ of the fiber probe. A rigorous analysis of the modal amplitudes at the reference angle is given in Appendix A.

Using (A4), the nonzero transversal component of the electrical field $\left(E_{t}\right)$ in the MR can be expressed as

$$
\begin{aligned}
& E_{t}(r, \phi, z)=\sum_{q} \psi_{q}(r, z) G_{q}(r, \phi, z) \\
& G_{q}(r, \phi, z)=C_{q}^{(2)} \exp \left(-j \gamma_{q} \phi\right)+C_{q}^{(2)} \exp \left(j \gamma_{q} \phi\right)
\end{aligned}
$$

with $\psi_{q}$ as the transversal modal profile of WGM $P_{q}$.

The power collected by the fiber probe $P_{f}\left(r, \phi, d_{\text {tip }}\right)$ is proportional with the intensity at the aperture $I_{f}\left(r, \phi, d_{\mathrm{tip}}\right)$

$$
\begin{aligned}
P_{f}\left(r, \phi, d_{\mathrm{tip}}\right) \propto & I_{f}\left(r, \phi, z_{f}\right)=\left|E_{f}\left(r, \phi, d_{\mathrm{tip}}\right)\right|^{2} \\
I_{f}\left(r, \phi, d_{\mathrm{tip}}\right)= & \sum_{q}\left(I_{q}(\phi)+L_{q}(\phi)\right)\left|\psi_{q}\left(r, d_{\mathrm{tip}}\right)\right|^{2} \\
& +\sum_{q>p} M_{q, p}(\phi) \psi_{q}\left(r, d_{\mathrm{tip}}\right)\left(\psi_{p}\left(r, d_{\mathrm{tip}}\right)\right)^{*} \\
& + \text { complex conjugate (c.c.). }
\end{aligned}
$$

A detailed analysis of (3) (see also Appendix A) reveals that $I_{f}$ can be rearranged as:

1) the modal power $I_{q}(\phi)$ of the individual clockwise- and counterclockwise-propagating WGMs $P_{q}$

$$
I_{q}=\left|C_{q}^{(2)}\right|^{2} \exp \left(-2 \gamma_{q}^{\prime \prime} \phi\right)+\left|C_{q}^{\prime(2)}\right|^{2} \exp \left(2 \gamma_{q}^{\prime \prime} \phi\right)
$$

2) cross products $L_{q}(\phi)$ as a result of the interference between clockwise- and counterclockwise-propagating WGMs $P_{q}$

$$
L_{q}=C_{q}^{(2)}\left(C_{q}^{(2)}\right)^{*} \exp \left(-j 2 \gamma_{q}^{\prime} \phi\right)+\text { c.c. }
$$


3) cross products $M_{q, p}(\phi)$ as a result of the interference between the WGMs $P_{q}$ and $P_{p}$ inside the MR

$$
\begin{aligned}
M_{q, p}= & C_{q}^{(2)}\left(C_{p}^{(2)}\right)^{*} \exp \left(-j\left(\gamma_{q}^{\prime}-\gamma_{p}^{\prime}\right) \phi\right) \\
& \times \exp \left(-\left(\gamma_{q}^{\prime \prime}+\gamma_{p}^{\prime \prime}\right) \phi\right) \\
& +C_{q}^{(2)}\left(C_{p}^{(2)}\right)^{*} \exp \left(-j\left(\gamma_{q}^{\prime}+\gamma_{p}^{\prime}\right) \phi\right) \\
& \times \exp \left(-\left(\gamma_{q}^{\prime \prime}-\gamma_{p}^{\prime \prime}\right) \phi\right) \\
& +C_{q}^{(2)}\left(C_{p}^{(2)}\right)^{*} \exp \left(+j\left(\gamma_{q}^{\prime}+\gamma_{p}^{\prime}\right) \phi\right) \\
& \times \exp \left(+\left(\gamma_{q}^{\prime \prime}-\gamma_{p}^{\prime \prime}\right) \phi\right) \\
& +C_{q}^{(2)}\left(C_{p}^{(2)}\right)^{*} \exp \left(+j\left(\gamma_{q}^{\prime}-\gamma_{p}^{\prime}\right) \phi\right) \\
& \times \exp \left(+\left(\gamma_{q}^{\prime \prime}+\gamma_{p}^{\prime \prime}\right) \phi\right) .
\end{aligned}
$$

From (5) and (6), it follows that the spatial-spectral period of the interference between two WGMs is a function of real angular propagation constants of the WGMs only.

\section{FSR and Finesse}

In general the real angular propagation constant $\left(\gamma_{q}^{\prime}\right)$ depends both explicitly and implicitly (materials and waveguide dispersion) on the wavelength $(\lambda)$

$$
\gamma_{q}^{\prime}(\lambda)=\frac{2 \pi R N_{q}(\lambda)}{\lambda}
$$

with $N_{q}$ as the effective index of WGM $P_{q}$, defined analogously to the effective index of an SW mode.

For a sufficiently small wavelength range around a central wavelength $\left(\lambda_{c}\right), \gamma_{q}^{\prime}$ varies linearly with $\lambda$. As a consequence, the phase shift per round-trip in the MR can be written as

$$
\varphi_{q}(\lambda)=\varphi_{q}\left(\lambda=\lambda_{c}\right)+2 \pi\left(\left.\frac{\partial \gamma_{q}^{\prime}}{\partial \lambda}\right|_{\lambda=\lambda_{c}}\right)\left(\lambda-\lambda_{c}\right) .
$$

Assuming, for the moment, no reflections of WGMs in the MR (i.e., $\Gamma_{q}=0$ ), we can remove the interdependence between clockwise- and counterclockwise-propagating WGMs

$$
\begin{aligned}
\left|C_{q}^{(2)}\right|^{2} & =\frac{H_{q}}{1+F_{q} \sin ^{2}\left(\frac{\varphi_{q}}{2}\right)}\left|A^{(1)}\right|^{2} \\
\left|C_{q}^{(1)}\right|^{2} & =\frac{H_{q}^{\prime}}{1+F_{q} \sin ^{2}\left(\frac{\varphi_{q}}{2}\right)}\left|A^{(1)}\right|^{2} \\
H_{q} & =\left(\frac{\left|\kappa_{q}\right|}{1-\left|X_{q}\right|}\right)^{2} \\
H_{q}^{\prime} & =\left(\frac{\left|r_{q}\right|}{1-\left|X_{q}\right|}\right)^{2} \\
F_{q} & =\frac{4\left|X_{q}\right|}{\left(1-\left|X_{q}\right|\right)^{2}} \\
X_{q} & \equiv t_{q} \exp \left(-2 \pi \gamma_{q}^{\prime \prime}\right) \\
\varphi_{q} & \equiv 2 \pi \gamma_{q}^{\prime}-\arg \left\{t_{q}\right\} .
\end{aligned}
$$

From (9), it can be seen that a WGM $P_{q}$ is in resonance for an integer $\varphi_{q} / 2 \pi$. Identifying the spectral distance between subse- quent resonance peaks with the free spectral range $\left(\mathrm{FSR}_{q}\right)$ of WGM $P_{q}$ implies that

$$
\left(\mathrm{FSR}_{q}\right)^{-1}=-\left.\frac{\partial \gamma_{q}^{\prime}}{\partial \lambda}\right|_{\lambda=\lambda_{c}} .
$$

There is a direct relation between $\mathrm{FSR}_{q}$ and the wavelength dependence of $\gamma_{q}^{\prime}$. In the case where the waveguide and materials dispersion can be ignored, the $\mathrm{FSR}_{q}$ is analogous to the well-known expression for the FSR of a Fabry-Perot interferometer

$$
\mathrm{FSR}_{q}=\frac{\lambda_{c}}{\gamma_{q}^{\prime}\left(\lambda_{c}\right)}=\frac{\lambda_{c}^{2}}{2 \pi R N_{q}\left(\lambda_{c}\right)}
$$

However, the waveguide dispersion in cylindrical MRs can be quite strong [18]; therefore, we use (10) instead of (11) throughout this paper.

Another important quantity is the finesse $\left(F_{q}\right)$ of a WGM $P_{q}$

$$
F_{q} \equiv \frac{\mathrm{FSR}_{q}}{\mathrm{FWHM}_{q}} .
$$

It relates the $\mathrm{FSR}_{q}$ with the full-width at half-maximum $\left(\mathrm{FWHM}_{q}\right)$ of the spectra of the individual WGMs and is a function of the fraction of the field remaining in the MR after one round-trip $\left(\left|X_{q}\right|\right)$. Using (9), we find

$$
F_{q}=\frac{\pi}{2}\left\{\arcsin \left(\frac{1-\left|X_{q}\right|}{2 \sqrt{\left|X_{q}\right|}}\right)\right\}^{-1} .
$$

From (13), it follows that the finesse decreases monotonously with decreasing $\left|X_{q}\right|$. For too high losses $\left(F_{q}<1\right)$, the spectra of the WGMs do not have a meaningful FWHM anymore $\left(F_{q} \geq\right.$ $1)$.

\section{Spatial Mode Beat}

On varying the angle of observation $(\phi)$ inside the MR for a fixed wavelength $(\lambda)$, an interference pattern between the WGMs (spatial mode beat) can be observed [see also Fig. 1(a)]. From (4) to (6), it follows that each combination of WGMs $P_{q}, P_{p}$ gives rise to interference patterns with spatial mode-beat periods $\Delta \phi_{q, p}^{(+)}(\lambda), \Delta \phi_{q, p}^{(-)}(\lambda)$ for codirectionally and contradirectionally propagating WGMs $P_{q}, P_{p}$ respectively

$$
\Delta \phi_{q, p}^{( \pm)}(\lambda)=\frac{2 \pi}{\left|\gamma_{q}^{\prime}(\lambda) \mp \gamma_{p}^{\prime}(\lambda)\right|}
$$

From (14), it follows that it is sufficient to know the real part of the angular propagation constants of the WGMs in order to obtain the spatial mode-beat periods.

\section{E. Spectral Mode Beat}

Consider the case, without loss of generality, of a fixed angle of observation while varying the wavelength of the coupled light. An interference pattern similar to the spatial mode beat results, but now as a function of the wavelength (see also Fig. 2), the spectral mode beat. For analysis of the spectral mode beat, it is convenient to distinguish between the 
different contributions in the modal amplitudes corresponding to different numbers of round-trips $(m=0-\infty)$, such as

$$
\frac{1}{1-X_{q} \exp \left(-j \gamma_{q}^{\prime} 2 \pi\right)}=\sum_{m=0}^{\infty} X_{q}^{m} \exp \left(-j \gamma_{q}^{\prime} 2 \pi m\right)
$$

Inserting (15) and (A4) into (5) and (6) and separating into terms with common wavelength dependence gives

$$
\begin{aligned}
M_{q, p} & =\sum_{\nu_{q}, \nu_{p}} M_{ \pm q, \pm p}^{\left(\nu_{q}, \nu_{p}\right)} \\
M_{ \pm q, \pm p}^{\left(\nu_{q}, \nu_{p}\right)} & \propto \exp \left(j K_{ \pm q, \pm p}^{\left(\nu_{q}, \nu_{p}\right)}\left(\lambda-\lambda_{c}\right)\right), \\
\nu_{q}, \nu_{p} & \in\{0,1,2, \ldots\}
\end{aligned}
$$

where $K_{ \pm q, \pm p}^{\left(\nu_{q}, \nu_{p}\right)}$ is the spectral frequency of the spectral mode beat between WGMs $P_{q}$ and $P_{p}$, having propagated $\nu_{q}$ and $\nu_{p}$ round-trips in the $\mathrm{MR}$, and the subscripts - and + indicate clockwise and counterclockwise propagating WGMs.

As in Section III-B, three cases can be discerned:

1) the interference between counterclockwise-propagating WGMs $P_{q}$ and $P_{p}$

with spectral mode-beat period

$$
K_{q, p}^{\left(\nu_{q}, \nu_{p}\right)}=\frac{\phi+2 \pi \nu_{q}}{\mathrm{FSR}_{q}}-\frac{\phi+2 \pi \nu_{p}}{\mathrm{FSR}_{p}}
$$

$$
\Delta \lambda_{q, p}^{\left(\nu_{q}, \nu_{p}\right)}=\left|\frac{2 \pi}{K_{q, p}^{\left(\nu_{q}, \nu_{p}\right)}}\right|
$$

2) the interference between clockwise-propagating WGMs $P_{q}$ and $P_{p}$

with spectral mode-beat period

$$
K_{-q,-p}^{\left(\nu_{q}, \nu_{p}\right)}=\frac{\phi-2 \pi \nu_{q}}{\mathrm{FSR}_{q}}-\frac{\phi-2 \pi \nu_{p}}{\mathrm{FSR}_{p}}
$$

$$
\Delta \lambda_{-q,-p}^{\left(\nu_{q}, \nu_{p}\right)}=\left|\frac{2 \pi}{K_{-q,-p}^{\left(\nu_{q}, \nu_{p}\right)}}\right|
$$

3) the interference between contradirectionally propagating WGMs $P_{q}$ and $P_{p}$

$$
K_{q,-p}^{\left(\nu_{q}, \nu_{p}\right)}=\frac{\phi+2 \pi \nu_{q}}{\mathrm{FSR}_{q}}+\frac{\phi-2 \pi \nu_{p}}{\mathrm{FSR}_{p}}
$$

with spectral mode-beat period

$$
\Delta \lambda_{q,-p}^{\left(\nu_{q}, \nu_{p}\right)}=\left|\frac{2 \pi}{K_{q,-p}^{\left(\nu_{q}, \nu_{p}\right)}}\right|
$$

In order to calculate the spectral mode-beat periods for given orders $\nu_{p}$ and $\nu_{q}$, it is sufficient to know the FSRs of the WGMs involved.
Following the approach of this section, we can also find, alternative to (9), expressions for the intensity of the individual WGMs. By inserting (15) into (9) and collecting terms with common wavelength dependence, we find

$$
\begin{aligned}
& \left|C_{q}^{(2)}\right|^{2}=\sum_{\rho=-\infty}^{\infty} \hat{I}_{q}(\rho) \exp \left(j K_{q, q} \rho\left(\lambda-\lambda_{c}\right)\right) \\
& \left|C_{q}^{(1)}\right|^{2}=\sum_{\rho=-\infty}^{\infty} \hat{I}_{q}^{\prime}(\rho) \exp \left(j K_{q, q} \rho\left(\lambda-\lambda_{c}\right)\right)
\end{aligned}
$$

with

$$
\begin{aligned}
\hat{I}_{q}(\rho) & =\left|\kappa_{q} A^{(1)}\right|^{2} \exp \left(-j \rho \varphi_{q}\left(\lambda=\lambda_{c}\right)\right) \frac{\left|X_{q}\right| \rho \mid}{1-\left|X_{q}\right|^{2}} \\
\hat{I}_{q}^{\prime}(\rho) & =\left|r_{q} A^{(1)}\right|^{2} \exp \left(-j \rho \varphi_{q}\left(\lambda=\lambda_{c}\right)\right) \frac{\left|X_{q}\right|^{|\rho|}}{1-\left|X_{q}\right|^{2}} \\
K_{q, q} & =\frac{2 \pi}{\mathrm{FSR}_{q}}
\end{aligned}
$$

From (20), it can be concluded that the wavelength dependence of the intensity of the individual WGMs $P_{q}$ is a Fourier series with principle spectral frequency $K_{q, q}$. By a closer examination of (20), it follows that the magnitudes of the Fourier components $\left(\left|\hat{I}_{q}\right|,\left|\hat{I}_{q}^{\prime}\right|\right)$ of subsequent orders $( \pm|\rho|, \pm(|\rho|+1))$ differ only by a factor of $\left|X_{q}\right|$. This opens the way to extract $\left|X_{q}\right|$ and, via (13), the finesse from the Fourier transformation (with respect to the wavelength) of a wavelength scan.

\section{F. Estimating the Magnitudes of the Intracavity Phenomena}

In Sections III-A-E, it was shown that, for a global description of the intracavity behavior, it is sufficient to know the real angular propagation constants at the central wavelength and the FSRs of the WGMs involved. The magnitudes of the intracavity phenomena are, however, determined by the losses in the MR, the coupling with the SW, and the generation of contradirectionally propagating WGMs inside the MR. In this section, estimates for the magnitudes of the intracavity phenomena are given without a priori knowledge of the coupling with the SW and contradirectional coupling inside the MR.

First, consider the intensity of the individual WGMs $P_{q}$. In order to remove the interdependence between clockwise- and counterclockwise-propagating WGMs we assume, like in Section III-C, no reflections of the WGMs in the MR. Assuming lossless coupling between the WGMs and the SW mode, it can be shown that the upper limit for the modal intracavity power of a counterclockwise propagating WGM $P_{q}$ corresponds with impedance-matched coupling (i.e., losses per round-trip in the MR are balanced by the coupling with the SW)

$$
\begin{aligned}
\left|C_{q}^{(2)}\right|^{2} & \leq H_{q}^{(\mathrm{imp})}=\frac{1}{1-\left|X_{q}^{(0)}\right|^{2}} \\
\left|\kappa_{q}\right| & =\left|\kappa_{q}^{\prime}\right|=\left|\kappa_{q}^{(\mathrm{imp})}\right| \equiv \sqrt{1-\left|X_{q}^{(0)}\right|^{2}} \\
\left|X_{q}^{(0)}\right| & \equiv \exp \left(-2 \pi \gamma_{q}^{\prime \prime}\right) .
\end{aligned}
$$




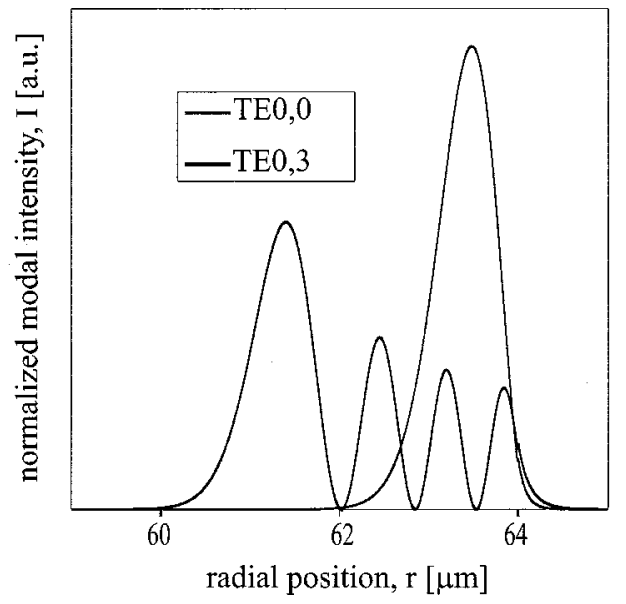

Fig. 4. Calculated (one-dimensional) transversal normalized modal intensities of the TE0,0 and TE0,3 WGMs.

With $\left|X_{q}^{(0)}\right|$ referring to the fraction of the modal amplitude remaining in a stand-alone MR (no coupling with the SW mode) after a single round-trip.

Analogous to (13), the unloaded (i.e., a stand-alone MR) finesse $\left(F_{q}^{(0)}\right)$ of a WGM $P_{q}$ can be defined

$$
F_{q}^{(0)}=\frac{\pi}{2}\left\{\arcsin \left(\frac{1-\left|X_{q}^{(0)}\right|}{2 \sqrt{\left|X_{q}^{(0)}\right|}}\right)\right\}^{-1} .
$$

The unloaded finesse of a WGM is an upper limit; as the finesse decreases monotonically with decreasing $\left|X_{q}\right|$, it follows that the coupling with the SW mode always results in a lowering of the finesse.

From (5) and (6), it can be concluded that the magnitudes of the spatial mode-beat phenomena are proportional with the product of the modal amplitudes inside the MR of the WGMs involved. As a consequence, (21) can be used for estimating the magnitude of a spatial mode-beat phenomenon.

In Section III-E, it was concluded that the spectral mode-beat periods depend on the number of round-trips of the WGMs in the MR. Moreover, it turns out that the magnitudes of the terms in (5) and (6) are proportional with the number of round-trips $(s)$ involved according to $\left|X_{q}\right|^{s}$. The magnitude of $\left|X_{q}\right|$ gives a direct estimate of the magnitude of a spectral mode-beat phenomenon in the intracavity intensity. Using $\left|X_{q}^{(0)}\right|$ of the WGMs involved, an upper boundary for the magnitude of the spectral mode beat can be given, without a priori knowledge of the coupling with the SW and contradirectional coupling inside the MR.

An intrinsic property of WGMs is that the positions of the principle maxima move toward the rim of the MR with decreasing radial order $l$; see Fig. 4 . As a consequence, the magnitude of an intracavity phenomenon also depends on the transversal modal profiles of the WGMs involved. In practice, the magnitude of the relevant products of transversal modal profiles $\left(\left|\psi_{q}\right|^{2}\right.$ for $I_{q}, L_{q}$ and $\left|\psi_{q} \psi_{p}\right|$ for $M_{q, p}$, respectively) at a position can be used to estimate the local magnitude of an intracavity phenomenon.

\section{ANALYSis OF A Highly Multimodal CylindRICAL MR AND COMPARISON WITH EXPERIMENTS}

The theory described in Section III is applied to a highly multimodal MR, which is a good test case for our model because it shows a rich variety of intracavity. Using detailed numerical methods, the wavelength dependence of the angular propagation constants $\left(\gamma_{q}(\lambda)\right)$ of the WGMs $P_{q}$ are calculated and the free spectral range, unloaded finesse, spatial mode-beat periods, and spectral frequencies of the spectral mode beat are calculated. The calculated parameters are compared with high-resolution PSTM measurements.

In all experiments, we excited a TE-polarized mode in the SW using a tunable dye laser and varying the wavelength $(\lambda=$ 648-678 nm). In all experiments, we checked the consistency between the measured spatial and spectral intensity distributions inside the MR and the measured power at the input and output ports of the MR. For more details on the experiments, the reader is referred to [8], [10], [11].

\section{A. MR Under Study}

The MR under study is realized in a low pressure chemical vapor deposition (LPCVD) $\mathrm{Si}_{3} \mathrm{~N}_{4}$ [22] (index $n \sim 2.01$ ) layer with thermal $\mathrm{SiO}_{2}(n \sim 1.458)$ lower cladding and air as upper cladding on a silicon substrate [see Fig. 3(b)]. The high (effective) index contrast MR is realized by means of reactive ion etching (RIE) and the low (effective) index contrast SW is realized by wet-chemical etching in a buffered hydrogen fluoride (B-HF) solution. By characterizing the MR for its functional behavior, an experimental value for the scattering and materials losses was obtained [2]: $\alpha_{\mathrm{SC}} \sim 77.5 \mathrm{~dB} \cdot \mathrm{cm}^{-1}$. Throughout this paper, it is assumed that $\alpha_{\mathrm{sc}}$ is identical for all WGMs.

\section{B. Calculation of the Angular Propagation Constant and Losses in the MR}

In all of our calculations, we started by reducing the three-dimensional (3-D) problem into a two-dimensional (2-D) problem by means of the well-known effective index method (EIM). By conformal mapping [23], it is possible to transform a curved 2-D waveguide into a SW with a transformed index profile. The equivalent SW problem was solved using the Wentzel-Kramers-Brillouin (WKB) approximation technique [23], [24]. We checked our results by directly solving the (2-D-3-D) Maxwell's equations in cylindrical coordinates (e.g., finite-difference methods [25]) in combination with perfectly matched layer (PML) boundary conditions [26]). The resulting real angular propagation constants agree well (within 1\%) with the results of directly solving the (2-D-3-D) Maxwell's equations in polar-cylindrical coordinates. Fitting (8) to the wavelength dependence of the real angular propagation constants, the FSRs of the WGMs follow naturally (see Table I). The calculated $\gamma_{q}$ decrease and FSRs increase for increasing radial order $l$. In Table I, the wavenumber $\left(K_{q, q}\right)$ corresponding with the FSR is given. By using the propagation losses, the fraction remaining in a stand-alone MR after one round-trip $X_{q}^{(0)}$, the unloaded finesse $F^{(0)}$, and the upper limit for the modal intracavity power $H_{q}^{(\mathrm{imp})}$ have been calculated. 
TABLE I

Calculated Angular Propagation Constants $\left(\gamma^{\prime}\right)$, Propagation losses $\left(\alpha_{\text {prop }}\right)$, and FSRs of the WGMs Around a Central WAVELENGTH $\lambda_{c}=663.8 \mathrm{~nm}$

\begin{tabular}{|c|c|c|c|c|c|c|c|}
\hline WGM & $\begin{array}{c}\gamma^{\prime} \\
{\left[\mathbf{r a d}^{-1}\right]}\end{array}$ & $\begin{array}{c}\alpha_{\text {prop }} \\
{\left[\mathrm{dB} \cdot \mathrm{cm}^{-1}\right]}\end{array}$ & $\begin{array}{l}\text { FSR } \\
\text { [pm] }\end{array}$ & $\begin{array}{r}\mathbf{K}_{\mathrm{q}, \mathrm{q}} \\
{\left[\mathbf{n m}^{-1}\right]}\end{array}$ & $\begin{array}{l}\mathbf{X}_{\mathrm{q}}{ }^{(0)} \\
{[\%]}\end{array}$ & $F^{(0)}$ & $\mathbf{H}_{\mathrm{q}}{ }^{\left({ }^{(i m p)}\right.}$ \\
\hline $\mathrm{E} 0,0$ & 1109.6 & 77.5 & 533 & 11.8 & 69.9 & 8.7 & 1.95 \\
\hline 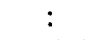 & & & & & 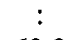 & : & \\
\hline TE0,4 & 1065.4 & 79.3 & 540 & 11.6 & 69.3 & 8.5 & 1.92 \\
\hline M0,0 & 1065.0 & 77.5 & 514 & 12.2 & 69.9 & 8.7 & 1.95 \\
\hline : & : & : & & & : & 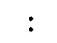 & \\
\hline TM0,4 & 1020.5 & 77.5 & 522 & 12.0 & 69.9 & 8.7 & 1.95 \\
\hline $\mathrm{TE} 1,0$ & 874.8 & 950.5 & 584 & 10.8 & 1.2 & $\ldots$ & 1.00 \\
\hline
\end{tabular}

The bending losses $\left(\alpha_{\text {bend }}\right)$ in the MR were calculated by directly solving Maxwell's equations. By adding the scattering and material losses to the bending losses, the propagation losses $\left(\alpha_{\text {prop }}=\alpha_{\text {bend }}+\alpha_{\mathrm{sc}}\right)$ and the unloaded finesses of the WGMs were calculated (see Table I), as well. As the bending losses increase for increasing radial order $l$, it follows that $\left|X_{q}^{(0)}\right|$, and, consequently, $H_{q}^{(\mathrm{imp})}$ and the unloaded finesse decrease for increasing $l$.

\section{Relevant WGMs}

Because the MR is highly multimodal, it is important to reduce the number of WGMs included in our calculations in order to keep the analysis traceable. The relevant WGMs can be selected by considering the magnitudes of $H_{q}^{(\mathrm{imp})}$. From Table I, it can be concluded that the modal amplitudes of the WGMs are, at the most, somewhat larger than the modal intensity to the left of the MR $\left(\left|A^{(1)}\right|^{2}\right)$ of the SW mode. The magnitudes of $H_{q}^{\text {(imp) }}$ of the WGMs supported by the MR are of the same order of magnitude and, as a consequence, by just considering the upper limits of the modal amplitudes, it is not possible to reduce the set of relevant WGMs. Because $H_{q}^{(\mathrm{imp})}$ is an upper boundary for the modal power, one actually needs to include the coupling between the SW mode and the WGMs. The TE1,0 WGM is close to cutoff and, consequently, has a strong overlap (i.e., coupling) with the SW mode. Even though the losses of the TE1,0 WGM are rather high (i.e., $\alpha \sim 9.6 \mathrm{~dB} / 90^{\circ}$ ), they can be offset by a strong coupling of the TE1,0 WGM with the SW mode. Therefore, we have decided to include the TE1,0 WGM in our analysis.

By limiting the study of the MR to a small region (with respect to the radial coordinate $r$ ) of the MR, one can further reduce the set of WGMs used in the analysis. Within the scope of this paper, we will limit the analysis to $r \geq 60 \mu \mathrm{m}$, which comprises the principle maxima of the TE0,0-TE0,3; TM0,0-TM0,3; and TE1,0 WGMs. Taking into account that $\left|M_{q, p}\right|$ scales linearly with $\left|\psi_{q} \psi_{p}\right|$, it follows that the largest cross products due to the mode beat can be expected from WGMs with a considerable overlap of the modal profiles (i.e., WGMs with subsequent radial orders $l$ ). In order to assure that the interference of the TE0,3-TM0,3 modes with the other WGMs is described adequately, without drastically increasing the set of modes, the TE0,4-TM0,4 WGMs have been included in the analysis, as well.

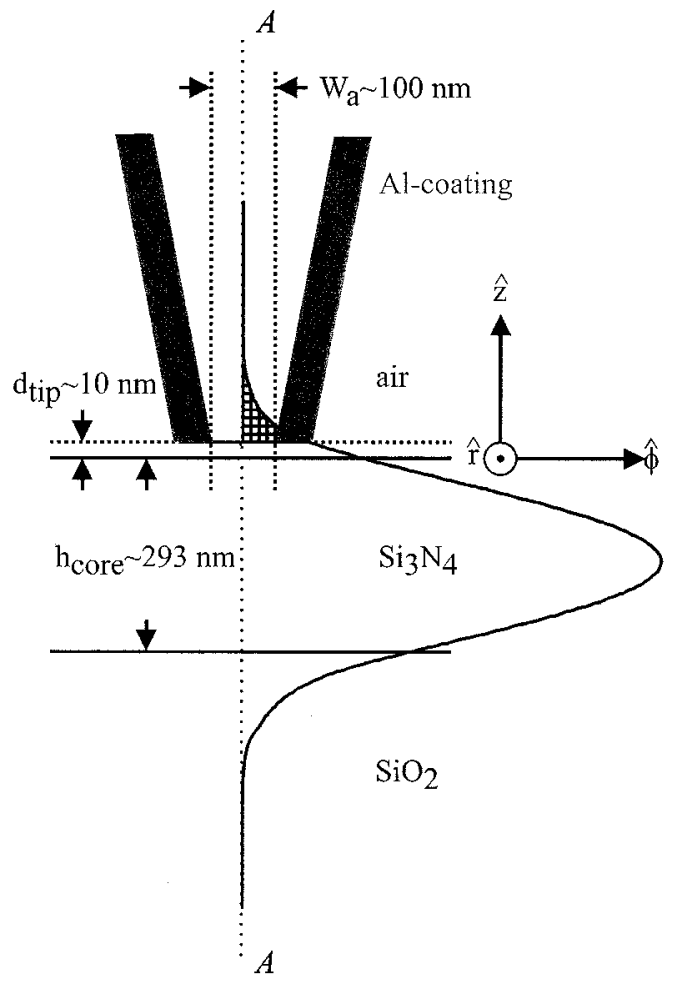

Fig. 5. Schematic picture of an Al-coated fiber tip that probes the evanescent field of a cylindrical MR. The shaded area represents the modal overlap between the fiber probe and the intracavity intensity of the MR.

\section{Influence of the Fiber Tip of the PSTM}

Our PSTM [11] (see also Fig. 5) has an aluminum-coated tapered $\left(\theta_{\text {tap }} \sim 15^{\circ}\right)$ fiber tip with an aperture $W_{a} \sim 100 \mathrm{~nm}$. The distance between the fiber tip of the PSTM and the air$\mathrm{Si}_{3} \mathrm{~N}_{4}$ interface was kept constant at $d_{\mathrm{tip}} \sim 10 \mathrm{~nm}$. In the analysis of the intracavity intensity, we ignored the influence of the fiber tip. The influence of the fiber tip on the intracavity field can be estimated by calculating the fraction $\left(X_{\text {tip }}\right)$ of the modal amplitude remaining in the WGM after having passed the fiber tip. The presence of the fiber tip modifies the fraction of the modal amplitude of a WGM remaining in the MR after one round-trip $\left(X_{q}\right)$

$$
X_{q} \rightarrow X_{q} \cdot X_{\text {tip }}
$$

Because we are mainly interested in an upper limit for the influence of the fiber tip on the intracavity intensity, we assume that the width of the fiber tip is larger than the modal width of 
TABLE II

Comparison BetweEn the Calculated and ObSERVEd Spatial MODE-BEAT LENGTHS $\Delta s^{(+)}[\mu \mathrm{m}]$ AND $\Delta s_{c}^{(-)}[\mu \mathrm{m}]$ OF CODIRECTIONALLY AND CONTRADIRECTIONALLY PROPAGATING WGMS $P_{q}$ AND $P_{p}$

\begin{tabular}{|c|c|c|c|c|}
\hline$\overline{\text { type }}$ & $\begin{array}{l}\text { WGM } \\
P_{q}\end{array}$ & $\begin{array}{l}\text { WGM } \\
\mathbf{P}_{\mathrm{p}}\end{array}$ & Calculated & Observed \\
\hline \multirow{6}{*}{$\Delta s_{\mathrm{q}, \mathrm{p}}^{(+)}$} & $\begin{array}{l}\text { TE0,0 } \\
\text { TM0,0 }\end{array}$ & $\begin{array}{l}\text { TE0,1 } \\
\text { TM0,1 }\end{array}$ & $\begin{array}{l}29.1 \\
28.9\end{array}$ & $29 \pm 14$ \\
\hline & TE0,1 & TM0,0 & 13.1 & $14 \pm 2$ \\
\hline & TE0,1 & TM0,1 & 9.0 & $10 \pm 2$ \\
\hline & TE 0,0 & TM0,1 & 6.9 & $7.2 \pm 0.9$ \\
\hline & $\mathrm{TE} 0,0$ & TM0,2 & 5.7 & $5.7 \pm 0.6$ \\
\hline & TE1,0 & $\begin{array}{l}\text { TE0, } 1^{\prime} \\
\text { TM0, } 1^{\prime}\end{array}$ & $\begin{array}{l}1.7-2.1 \\
2.1-2.8\end{array}$ & $(2.1-2.8) \pm 0.1$ \\
\hline \multirow{4}{*}{$\Delta s_{q, p}^{(-)}$} & TE0,1 & TE0,1' & $0.181-0.189$ & $0.177 \pm 2 \cdot 10^{-3}$ \\
\hline & TM0,1 & TM0, $1^{\prime}$ & $0.189-0.197$ & $0.197 \pm 2 \cdot 10^{-3}$ \\
\hline & TE1,0 & $\begin{array}{l}\text { TE0, } \mathbf{1}^{\prime} \\
\text { TM0, } \mathbf{l}^{\prime}\end{array}$ & $0.203-0.212$ & $0.201 \pm 2 \cdot 10^{-3}$ \\
\hline & TE1,0 & TE 1,0 & 0.230 & $0.232 \pm 3 \cdot 10^{-3}$ \\
\hline
\end{tabular}

the WGMs and, therefore, approximate the MR with the fiber tip on top by an equivalent slab waveguide, like in cross section $A-A$ in Fig. 5. From detailed numerical simulations using a 2-D finite difference beam propagation method (FD-BPM), we could estimate $X_{\text {tip }}$ for the TE $k(k=0,1)$ and $\operatorname{TM} k(k=0)$ modes of the equivalent slab waveguide. Because $X_{\text {tip }} \sim 1$ (i.e., $X_{\text {tip }} \sim 99.2 \%$ for the TE0 and $X_{\text {tip }} \sim 97.5 \%$ for the TM0 mode) and the losses per round-trip of the $k=0$ WGMs are already quite large, we do not expect a large influence of the fiber tip on the resonance-characteristics (i.e., finesse) and modal amplitudes of these WGMs. For the TE1,0 WGM, a somewhat larger decrease of the modal amplitude due to the presence of the fiber tip is expected ( $X_{\text {tip }} \sim 43.5 \%$ ), but because the WGM hardly completes a single round-trip $\left(\left|X_{q}^{(0)}\right| \sim 1.2 \%\right)$, no large influence of the fiber tip on the resonance-characteristics of the TE1,0 WGM is expected. Therefore, we conclude that the presence of the fiber tip is not expected to have a large influence on the intracavity intensity of the MR under study, because the WGMs have already relatively high losses per round-trip in the MR. For high-finesse WGMs, the presence of the fiber tip can result in a significant decrease of the unloaded finesse and cannot, therefore, be ignored in the case of weak coupling with the SW mode. For a more detailed study of the influence of the fiber tip on the intensity, which is outside the scope of this paper, the reader is referred to the computational study in [27] on the influence of the fiber tip on the intensity inside a photonic crystal.

\section{E. Spatial Mode Beat}

For the comparison with the experimental results, it is convenient to transform $\Delta \phi_{q, p}$ into a mode-beat length (corresponding to the distance along the rim of the MR)

$$
\Delta s_{q, p}^{( \pm)}(\lambda) \equiv R \cdot \Delta \phi_{q, p}^{( \pm)}(\lambda) .
$$

The calculated mode-beat lengths (see also Table II) between codirectionally propagating WGMs range from $\Delta s_{q, p}^{(+)}(\lambda)=$

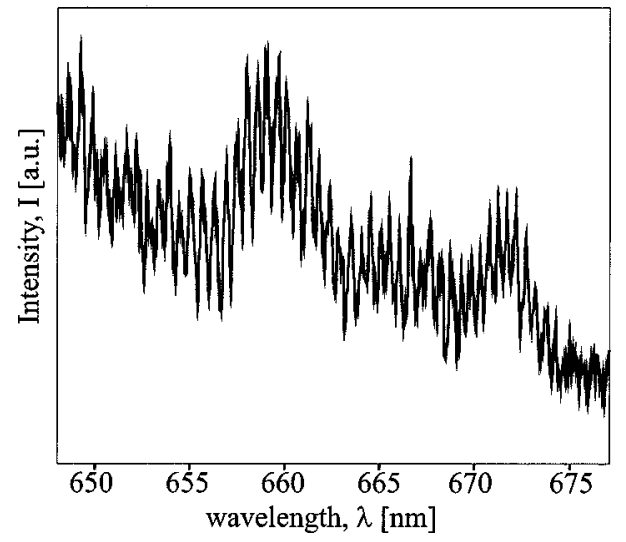

Fig. 6. Measured wavelength line scan of the intracavity intensity at an angle of observation of $90^{\circ}$ close to the rim of the MR.

1.7-941.7 $\mu \mathrm{m}$ and $\Delta s_{q, p}^{(-)}(\lambda)=181-230 \mathrm{~nm}$ for the contradirectionally propagating WGMs, respectively.

By mapping the intensity inside the MR at a fixed wavelength, we extracted a variety of spatial mode-beat lengths (see Table II). It should be noted that, by this method, only spatial mode-beat lengths smaller than half the circumference of the MR can be observed, $\Delta s \leq 201 \mu \mathrm{m}$. From comparing these spatial mode-beat lengths with the theoretical results (see Table II), we could assign the experimental spatial mode-beat lengths to combinations of WGMs. The observation of spatial mode beat between codirectionally propagating TE- and TM-polarized WGMs indicates polarization conversion by the MR, because the SW-mode was TE polarized. The observed subwavelength spatial mode-beat periods give unambiguous evidence of the presence of contradirectionally propagating WGMs and also illustrate the optical resolution of the PSTM $(\Delta x \leq 50 \mathrm{~nm})$. We observed relatively deep modulations [11] in the intensity due to the co- and contradirectional mode beat involving the TE1,0 WGM, which indicates a strong excitation of the TE1,0 WGM despite of the high-propagation losses of this WGM.

\section{F. FSR and Finesse of the WGMs}

By mapping the intensity inside the MR at a fixed angle of observation $\left(\phi=90^{\circ}\right)$ while varying the wavelength and radial position $(r)$ (spatiospectral scan, see also Fig. 2), we obtained detailed information about the spatial and spectral characteristics of the intensity distribution. From the wavelength line scan in Fig. 6, it follows immediately that the intensity as a function of the wavelength varies rapidly with a period around $0.5 \mathrm{~nm} \mathrm{su}-$ perposed on a slowly varying modulation. Applying a Fourier transform (with respect to the wavelength) to the wavelength scan (see Fig. 6), it is possible to extract the spectral frequencies $K$ of the individual WGMs and the mode beat between them.

As a result of the limited scan range (i.e., $\lambda=648-678 \mathrm{~nm}$ resulting in a spectral frequency resolution of $\Delta k \sim 0.22 \mathrm{~nm}^{-1}$ ), we could not distinguish between peaks corresponding to the FSRs of different radial-order WGMs (i.e., the spacing between the FSRs of WGMs of subsequent radial order is around $2 \mathrm{pm}$ ). In the spectral frequency interval $K=10.6-12.4 \mathrm{~nm}^{-1}$ (see bar I in Fig. 7), we observed strong peaks corresponding to the 


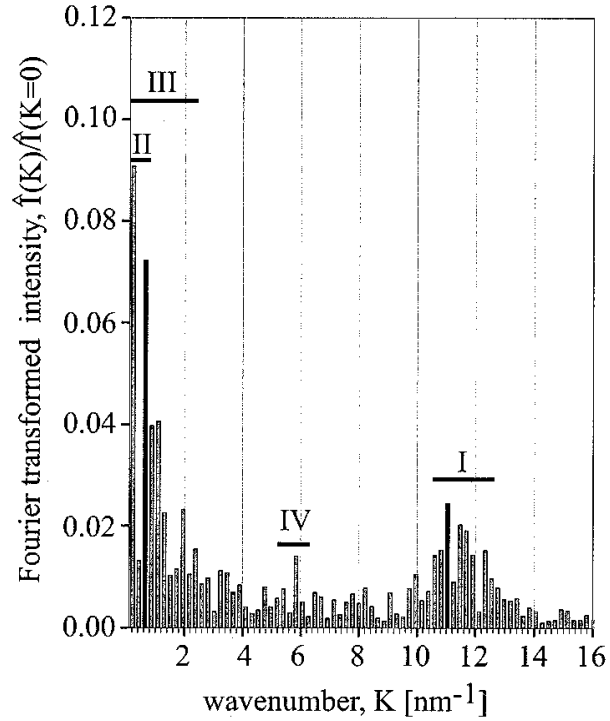

Fig. 7. Fourier transform of the wavelength scan in Fig. 6 normalized by its direct current component (i.e., wavenumber $K=0 \mathrm{~nm}^{-1}$ ). The horizontal bars indicate the wavenumber intervals as referred to in Table III.

principal spectral frequency $K_{q, q}$ (i.e., $\rho=1$ ) of the individual WGMs, as predicted by our calculations (see Table I). The strongest (black) peak could be assigned to the TE1,0 WGM, which indicates (as seen in in the Section IV-E) a strong excitation of this WGM. Because no clear peaks in the spectral frequency interval corresponding to $\rho \geq 2$ could be observed, we conclude that the finesse of the WGMs is limited to $F<2$, which is attributed to the high scattering and materials losses, and for the TE1,0 WGM, to the high bending losses.

\section{G. Spectral Mode Beat}

In Section III-F, it was concluded that the magnitude of the spectral mode beat between the WGMs $P_{p}$ and $P_{q}$ of orders $\nu_{p}$ and $\nu_{q}$ is proportional with

$$
\left|M_{q, p}^{\nu_{q}, \nu_{p}}\right| \propto\left|X_{q}\right|^{\nu_{q}} \cdot\left|X_{p}\right|^{\nu_{p}} \leq\left|X_{q}^{(0)}\right|^{\nu_{q}} \cdot\left|X_{p}^{(0)}\right|^{\nu_{p}} .
$$

For the MR under study, $\left|X_{q}^{(0)}\right| \leq 69.9 \%$ for all WGMs $P_{q}$ (see Table I), implying that the magnitudes of the higher order $\left(\nu_{p}+\nu_{q}>0\right)$ spectral mode-beat phenomena are significantly smaller than the magnitudes of the fundamental $\left(\nu_{p}+\nu_{q}=0\right)$ spectral mode beat. Therefore, we have decided to focus the analysis on the fundamental spectral mode beat.

The calculated spectral mode-beat periods for codirectionally and contradirectionally propagating WGMs at an angle of observation $\phi=90^{\circ}$ can be found in Table III and the horizontal bars in Fig. 7 indicate a range of spectral frequencies $K$ that correspond with a spectral mode-beat phenomenon. Bars I-III could be assigned to the spectral mode beat between codirectionally propagating WGMs. In Fig. 7, a broad band of spectral frequencies indicated by bar I somewhat obscures the presence of peaks at the spectral frequencies of the individual WGMs. Bar IV indicates the presence of fundamental spectral mode beat between contradirectionally propagating WGMs, which confirms the previous observation of contradirectionally propagating WGMs in the analysis of the spatial mode beat. The fact that no higher order spectral mode beat between
TABLE III

CALCUlated WaVENumbers AND CORRESPONDING EXPERIMENTAl BARS (as Indicated in Fig. 7) OF THE SPectral Mode Beat Between CODIRECTIONALLY COUNTERCLOCKWISE $\left(K_{q, p}^{\left(\nu, \nu_{p}\right)}\left[\mathrm{nm}^{-1}\right]\right)$, CLOCKWISE $\left(K_{-q, p}^{\left(\nu_{q}, \nu p\right.}\left[\mathrm{nm}^{-1}\right]\right)$, AND CONTRADIRECTIONALLY $\left(K_{q,-p}^{\left(\nu_{q}, \nu_{p}\right)}\left[\mathrm{nm}^{-1}\right]\right)$ PROPAGATING WGMS AT AN ANGLE OF OBSERVATION $\phi=90^{\circ}$

\begin{tabular}{|c|c|c|c|}
\hline \multicolumn{3}{|c|}{ Calculation } & Experiment \\
\hline Type & $\left(v_{p}, v_{q}\right)$ & $\mathrm{K}\left[\mathrm{nn}^{-1}\right]$ & Bar \\
\hline \multirow{3}{*}{$\mathrm{K}_{\mathrm{q}, \mathrm{p}}^{\left(v_{q}, v_{p}\right)}$} & $(0,0)$ & $<0.4$ & II \\
\hline & $(1,0)$ & $11.4-12.6$ & I \\
\hline & $(1,1)$ & $<1.8$ & III \\
\hline \multirow{3}{*}{$\mathbf{K}_{-q, p-p}^{\left(v_{q}, v_{p}\right)}$} & $(0,0)$ & $<0.4$ & II \\
\hline & $(1,0)$ & $11.4-12.2$ & I \\
\hline & $(1,1)$ & $<1.1$ & III \\
\hline \multirow{3}{*}{$\mathrm{K}_{\mathrm{q},-\mathrm{p}}^{\left(v_{q}, v_{\mathrm{p}}\right)}$} & $(0,0)$ & $5.4-6.1$ & IV \\
\hline & $(1,0)$ & $16.1-18.3$ & --- \\
\hline & $(1,1)$ & $5.3-7.2$ & --- \\
\hline
\end{tabular}

contradirectionally propagating WGMs was observed confirms the high propagation losses of the WGMs. At the right end of bar II, we observed a strong (black) peak, which could be assigned to the spectral mode beat between the codirectionally propagating TE1,0 and TM0,0 WGMs, once again indicating a strong excitation of the TE1,0 WGM.

\section{CONCLUSION}

We have presented a parametric model of the intracavity intensity inside a cylindrical MR with only the real angular propagation constants and FSRs as input. The model allows for a detailed analysis of intracavity phenomena, like spatial and spectral mode beat between WGMs in a cylindrical MR. With our model, we analyzed a highly multimodal cylindrical MR and evaluated the results by comparison with PSTM measurements of the intensity inside the MR. By analyzing spatial and spectral PSTM scans with our model, we were able to identify intracavity phenomena like polarization conversion and counterpropagation. The remarkably good agreement between the experimental results and our calculations shows the strength of the presented model for analyzing the intracavity phenomena inside cylindrical MRs.

\section{APPENDIX A \\ RIGOROUS ANALYSIS OF THE EXCITATION OF COUNTERLOCKWISE- AND CLOCKWISE-PROPAGATING WGMs}

The excitation of clockwise and counterclockwise WGMs by the coupling with the SW mode is discussed. Assuming no coupling between WGMs of different order $q$, it possible to describe the modal amplitudes of the clockwise- and counterclockwisepropagating WGMs [see also Fig. 3(a)] by

$$
\begin{aligned}
C_{q}^{(2)} & =t_{q} C_{q}^{(1)}+\Gamma_{q} C_{q}^{(2)}-j \kappa_{q} A^{(1)}+r_{q} A^{(2)} \\
C_{q}^{(1)} & =\exp \left(-j \gamma_{q} 2 \pi\right) C_{q}^{(2)} \\
C_{q}^{(1)} & =\Gamma_{q} C_{q}^{(1)}+t_{q} C_{q}^{(2)}+r_{q} A^{(1)}-j \kappa_{q} A^{(2)} \\
C_{q}^{(2)} & =\exp \left(-j \gamma_{q} 2 \pi\right) C_{q}^{(1)} .
\end{aligned}
$$


After some trivial manipulations, it is possible to express the modal amplitudes of the WGMs in terms of the modal amplitudes in the SW

$$
\begin{aligned}
C_{q}^{(2)} \cdot N= & \left(1-t_{q} \exp \left(-j \gamma_{q} 2 \pi\right)\right) S+\Gamma_{q} \exp \left(-j \gamma_{q} 2 \pi\right) S^{\prime} \\
C_{q}^{\prime(2)} \cdot N= & \left(1-t_{q} \exp \left(-j \gamma_{q} 2 \pi\right)\right) \exp \left(-j \gamma_{q} 2 \pi\right) S^{\prime} \\
& +\Gamma_{q} \exp \left(-j \gamma_{q} 4 \pi\right) S
\end{aligned}
$$

with

$$
\begin{aligned}
N & =\left(1-t_{q} \exp \left(-j \gamma_{q} 2 \pi\right)\right)^{2}-\Gamma_{q}^{2} \exp \left(-j \gamma_{q} 4 \pi\right) \\
S & =-j \kappa_{q} A^{(1)}+r_{q} A^{(2)} \\
S^{\prime} & =-j \kappa_{q} A^{(2)}+r_{q} A^{(1)}
\end{aligned}
$$

In practice, both the evanescent field coupling coefficients between the SW modes and WGMs and the reflections at the reference angle are small $\left(\left|t_{q}\right| \gg\left|\Gamma_{q}\right|\right)$. As a consequence, $N$ in (A2) can be approximated by

$$
N \sim\left(1-X_{q} \exp \left(-j \gamma_{q}^{\prime} 2 \pi\right)\right)^{2}
$$

with

$$
X_{q} \equiv t_{q} \exp \left(-\gamma_{q}^{\prime \prime} 2 \pi\right) .
$$

Using (A3) and ignoring backreflections from the in-coupling and out-coupling facets (i.e., $A^{\prime(2)}=0$ ), it is possible to express the modal amplitudes of the WGMs in terms of the modal amplitude of the SW- mode (i.e., $A^{(1)}$ )

$$
\begin{aligned}
\frac{C_{q}^{(2)}}{A^{(1)}}= & \frac{1}{1-X_{q} \exp \left(-j \gamma_{q}^{\prime} 2 \pi\right)} \\
& \cdot\left(-j \kappa_{q}+\frac{B_{q}^{(2)}}{1-X_{q} \exp \left(-j \gamma_{q}^{\prime} 2 \pi\right)}\right) \\
\frac{C_{q}^{(2)}}{A^{(1)}}= & \frac{\exp \left(-\gamma_{q}^{\prime \prime} 2 \pi\right) \exp \left(-j \gamma_{q}^{\prime} 2 \pi\right)}{1-X_{q} \exp \left(-j \gamma_{q}^{\prime 2 \pi)}\right.} \\
& \cdot\left(r_{q}+\frac{B_{q}^{\prime(2)}}{1-X_{q} \exp \left(-j \gamma_{q}^{\prime} 2 \pi\right)}\right)
\end{aligned}
$$

with

$$
\begin{aligned}
B_{q}^{(2)} & =\Gamma_{q} r_{q} \exp \left(-\gamma_{q}^{\prime \prime} 2 \pi\right) \\
B_{q}^{\prime(2)} & =-j \kappa_{q} \Gamma_{q} \exp \left(-\gamma_{q}^{\prime \prime} 2 \pi\right) .
\end{aligned}
$$

\section{ACKNOWLEDGMENT}

The authors wish to thank Kymata Software for use of its numerical modesolving software, Tempselene Pro 4.2.

\section{REFERENCES}

[1] B. E. Little, S. T. Chu, H. A. Haus, J. Foresi, and J. P. Laine, "Microring resonator channel dropping filters," J. Lightwave Technol, , vol. 16, pp. 998-1005, June 1997.

[2] F. C. Blom, D. R. van Dijk, H. J. W. M. Hoekstra, A. Driessen, T. J. A. Popma, S. T. Chu, and B. E. Little, "A single channel dropping filter based on a cylindrical microresonator," Opt. Commun., vol. 167, pp. $77-82,1999$.

[3] C. Gmachl, F. Capasso, E. E. Narimanov, J. U. Nöckel, A. D. D. Stone, J. Faist, D. L. Sivco, and A. Y. Cho, "High power directional emission from microlasers with chaotic resonators," Science, vol. 280, pp. 1557-1564, 1998.

[4] A. F. J. Levi, R. E. Slusher, S. L. McCall, J. L. Glass, S. J. Pearton, and R. A. Logan, "Directional light coupling from microdisk laser," Appl. Phys. Lett., vol. 62, pp. 561-563, 1993.
[5] F. C. Blom, H. Kelderman, H. J. W. M. Hoekstra, A. Driessen, and T. J. A. Popma, "Experimental study of integrated optics microcavity resonators: Toward and all-optical switching device," Appl. Phys. Lett., vol. 71, pp. 747-749, 1997.

[6] B. E. Little, S. T. Chu, W. Pan, and Y. Kokubun, "Microring resonators arrays for VLSI photonics," IEEE Photon. Technol. Lett., vol. 12, pp. 323-325, Mar. 2000.

[7] D. Rafizadeh, J. P. Zhang, R. C. Tiberio, and S. T. Ho, "Propagation loss measurements in semiconductor microcavity ring and disk resonators," J. Lightwave Technol., vol. 16, pp. 1308-1314, July 1998.

[8] M. L. M. Balistreri, D. J. W. Klunder, F. C. Blom, A. Driessen, H. J. W. M. Hoekstra, J. P. Korterik, L. Kuipers, and N. F. van Hulst, "Visualizing the whispering gallery modes in a cylindrical microcavity," Opt. Lett., vol. 24, pp. 1829-1831, 1999.

[9] G. H. Vander Rhodes, B. B. Goldberg, M. S. S. Ünlü, S. T. Chu, and B. E. Little, "Internal spatial modes in glass microring resonators," IEEE J. Select. Topics Quantum Electron., vol. 6, pp. 46-53, Jan./Feb. 2000.

[10] D. J. W. Klunder, M. L. M. Balistreri, F. C. Blom, H. J. W. M. Hoekstra, A. Driessen, L. Kuipers, and N. F. van Hulst, "High resolution photon scanning tunneling microscope measurements of the whispering gallery modes in a cylindrical microresonator," IEEE Photon. Technol. Lett., vol. 12, pp. 1531-1533, Nov. 2000.

[11] M. L. M. Balistreri, D. J. W. Klunder, F. C. Blom, A. Driessen, J. P. Korterik, L. Kuipers, and N. F. van Hulst, "Experimental analysis of the whispering gallery modes in a cylindrical optical microresonator," J. Opt. Soc. Amer. B, vol. 18, pp. 465-471, 2001.

[12] E. Betzig and J. K. Trautman, "Near-field optics: Microscopy, spectroscopy, and surface modifications beyond the diffraction limit," Science, vol. 257, pp. 2484-2486, 1992

[13] C. D. Poweleit, D. H. Naghski, S. M. Lindsay, J. T. Boyd, and H. E. Jackson, "Near field scanning optical microscopy measurements of optical intensity distributions in semiconductor channel waveguides," Appl. Phys. Lett., vol. 69, pp. 3471-3473, 1996.

[14] G. H. Vander Rhodes, B. B. Goldberg, M. S. Ünlü, S. T. Chu, W. P. T. Kaneko, Y. Kokobun, and B. E. Little, "Measurement of internal spatial modes and local propagation properties in optical waveguides," Appl. Phys. Lett., vol. 75, pp. 2368-2370, 1999.

[15] G. H. Vander Rhodes, M. S. Ünlü, J. M. Pomeroy, and T. F. Krauss, "Characterization of waveguide microcavities using high-resolution transmission spectroscopy and near-field scanning optical microscopy," IEE Proc. Optoelectron., vol. 145, pp. 379-383, 1998.

[16] S. Bourzeix, J. M. Moison, F. Mignard, F. Barthe, A. C. Boccara, C. Licoppe, B. Mersali, M. Allovon, and A. Bruno, "Near field optical imaging of light propagation in semiconductor waveguide structures," Appl. Phys. Lett., vol. 73, pp. 1035-1037, 1999.

[17] M. L. M. Balistreri, A. Driessen, J. P. Korterik, L. Kuipers, and N. F. van Hulst, "Quasi interference of perpendicularly polarized guided modes observed with a photon scanning tunneling microscope," Opt. Lett., vol. 25, pp. 637-639, 2000.

[18] S. C. Hagness, D. Rafizadeh, S. T. Ho, and A. Taflove, "FDTD microcavity simulations: Design and experimental realization of waveguide-coupled single-mode ring and whispering-gallery-mode disk resonators," J. Lightwave Technol., vol. 15, pp. 2154-2165, Nov. 1997.

[19] B. E. Little, J. P. Laine, and S. T. Chu, "Surface-roughness induced contradirectional coupling in ring and disk resonators," Opt. Lett., vol. 22, pp. 4-6, 1997.

[20] B. E. Little, S. T. Chu, and H. A. Haus, "Second-order filtering and sensing with partially coupled traveling waves in a single resonator," Opt. Lett., vol. 22, pp. 4-6, 1997.

[21] J. Capmany and M. A. Muriel, "A new transfer matrix formalism for the analysis of fiber ring resonators: Compound coupled structures for FDMA demultiplexing," J. Lightwave Technol., vol. 8, pp. 1904-1919, Dec. 1990.

[22] K. Wörhoff, P. V. Lambeck, and A. Driessen, "Design, tolerance analysis, and fabrication of silicon oxynitride based planar optical waveguides for communication devices," J. Lightwave Technol., vol. 17, pp. 1401-1407, Aug. 1999.

[23] M. Heiblum and J. H. Harris, "Analysis of curved optical waveguides by conformal transformation," IEEE J. Quantum Electron., vol. QE-11, pp. 75-83, 1975.

[24] E. C. M. Pennings, "Bends in Optical Waveguides (Modeling and Experiments)," Ph.D.dissertation, Delft University of Technology, Delft, The Netherlands, 1990

[25] W. W. Lui, C.-L. Xu, T. Hirono, K. Tokoyama, and W.-P. Huang, "Fullvectorial wave propagation in semiconductor optical bending waveguides and equivalent straight waveguide approximations," J. Lightwave Technol., vol. 16, pp. 910-914, May 1998. 
[26] W. P. Huang, C. L. Xu, W. Lui, and K. Yokoyama, “The perfectly matched layer boundary condition for modal analysis of optical waveguides: Leaky mode calculations," IEEE Photon. Technol. Lett. vol. 8, pp. 652-654, May 1996.

[27] S. Fan, I. Appelbaum, and J. D. Joannopoulos, "Near-field scanning optical microscopy as a simultaneous probe of fields and band structure of photonic crystals: A computational study," Appl. Phys. Lett., vol. 75, pp. 3461-3463, 1999

publication.
F. C. Blom, photograph and biography not available at the time of publication.

H. J. W. M. Hoekstra, photograph and biography not available at the time of publication.

A. Driessen (M'93-SM'95), photograph and biography not available at the time of publication.

L. Kuipers, photograph and biography not available at the time of publication.

N. F. van Hulst, photograph and biography not available at the time of publication. 